\title{
CORRECTIONS
}

\section{Between the Lines: Postponing grief}

In the print version (and the online pdf of the print version) of this article by Theodore Dalrymple, the picture we published was wrong (BMJ 2011;343:d5185, doi:10.1136/bmj.d5185).

The article was about Peter de Vries, but a mix-up in production led us to publish a picture of Mervyn Peake and not De Vries, as we said in the caption.

Cite this as: BMJ 2011;343:d5470

๑ BMJ Publishing Group Ltd 2011 